\title{
BARRIOS SALUDABLES
}

\author{
Ester Higueras García (Dra. Arquitecta - Profesora titular DUyOT)
}

Desde finales del siglo anterior, el desarrollo sostenible se ha mostrado como el camino necesario para afrontar los grandes retos de las ciudades. Con más de veinte años transcurridos desde la emblemática Cumbre de Rio de Janeiro (1992), en 2015 se pueden evaluar las fortalezas y debilidades que se han abierto con este nuevo objetivo global para ser aplicado desde las escalas locales. Un nuevo camino aparece con fuerza, el de los barrios saludables, entendiéndolos como una célula básica de sostenibilidad donde se pueden resolver satisfactoriamente gran cantidad de problemáticas sociales y ambientales ${ }^{23}$, como son la contaminación ambiental, el ruido urbano, la reducción del $\mathrm{CO}_{2}$, el aumento de las zonas verdes, el control microclimático de los espacios urbanos, el uso adecuado del espacio público y la reducción del stress urbano también necesario para la salud mental de todos los residentes. ${ }^{24}$

Aparece ahora como una línea de investigación prioritaria la transformación de los barrios de la ciudad consolidada en barrios saludables, y de esta forma afrontar algunos de los retos de sostenibilidad ambiental que debe resolver la ciudad del siglo $\mathrm{XXI}^{25}$. Por su peso cuantitativo en las ciudades actuales, las barriadas configuradas con las tipologías de la ciudad del movimiento moderno, se presentan como un gran espacio de oportunidad debido a su combinación de una alta densidad (en torres o edificaciones lineales), con alta proporción de espacios verdes, y con unas buenas condiciones teóricas de soleamiento y ventilación de todas la viviendas, pero que en la realidad necesitan un nuevo proyecto integrado y estructurado de sus espacios libres y zonas verdes. Esta tipología merece una reflexión detallada por diversas razones: en primer lugar, se trata de una tipología universal, es decir, se encuentra en las periferias de muchísimas ciudades europeas y americanas, debido a la adopción de una solución única en un momento en el que era necesaria la construcción masiva de viviendas para resolver la inmigración del campo a la ciudad; en segundo lugar los inmuebles son muy poco eficientes energéticamente hablando, debido a la falta casi unánime de sistemas de aislamiento y eficiencia en sus instalaciones, en este sentido han sido numerosos y sobresalientes los avances técnicos y constructivos para mejorar las condiciones de confort térmico de los inmuebles que se han producido desde los años 1970 hasta la actualidad y por tanto parece necesario y urgente introducir estos avances en esta tipología edificatoria para resolver los problemas de

\footnotetext{
23 http://www.euro.who.int/en/health-topics/environment-and-health/urban-health/activities/healthycities/who-european-healthy-cities-network/what-is-a-healthy-city. (Consultado dic 2014)

24 Destacan los proyectos de investigación financiados por fondos de la UE, en particular, INTARESE, HEIMTSA y PAREST, los tres de ellos proponen el Modelado de Evaluación Integrada para apoyar el Medio Ambiente de la UE y el Plan de Acción de Salud; el primero modela el riesgo de los factores de estrés ambiental, el segundo investiga sobre la salud y el impacto ambiental de los escenarios de las políticas urbanas, y el tercero, evalúa el impacto de la concentración de partículas contaminantes en suspensión (PM 10 y PM 2.5). También el proyecto INEQ-CITIES, cartografía las desigualdades socioeconómicas de la salud de las ciudades de la UE.

25 Acioly, C. (2000) 'Can Urban Management Deliver the Sustainable City?' en Compact Cities: Sustainable Urban Forms for Developing Countries, (eds.M.Jenks and R.Burgess), Spon Press, London.
} 
calidad del aire interior y confort térmico; en tercer lugar, en esta tipología se apuesta por soluciones constructivas universales ignorando completamente las consideraciones del lugar, o las lecciones aprendidas a lo largo del tiempo por edificaciones anteriores, con las aportaciones del urbanismo y la arquitectura bioclimática donde ya existe un amplio abanico de soluciones con muchas aplicaciones y mejoras para resolver los problemas específicos de estas edificaciones ${ }^{26}$; y por último aparece la oportunidad de mejorar las condiciones del microclima exterior y circundante a los edificios, con el convencimiento que si se mejoran la temperatura, humedad, viento y permeabilidad de los espacios exteriores urbanos, esto traerá una mejora en las condiciones del microclima exterior, atenuará los efectos negativos provocados por la isla térmica de calor urbano y mejorará las condiciones de los espacios exteriores para múltiples actividades.

En este sentido, la existencia de bajas ocupaciones de suelo con altas densidades edificatorias, resulta completamente oportuno para resolver algunas de las disfucionalidades térmicas y ambientales tanto de los espacios abiertos como de las edificaciones ${ }^{27}$. Este camino, se vislumbra como necesario y oportuno, y una vez adaptado a las condiciones generales del clima de cada ciudad ${ }^{28}$, puede seguirse para resolver este problema en numerosas ciudades americanas o europeas, y sin duda abre un cauce de acción necesario para afrontar la sostenibilidad de la ciudad consolidada, a través de acciones propositivas locales.

\section{Barrios saludables para una ciudad sostenible.}

Los barrios saludables, se entienden como la unidad desde la cual es posible afrontar y resolver algunos de los problemas de eficiencia y sostenibilidad ambiental que hoy día tienen planteadas la mayor parte de las ciudades del planeta, con un alcance desde la arquitectura y el urbanismo realista y propositivo. Las ciudades nunca fueron pensadas para que fueran eficientes. Es por esto, que desde 1980 donde los problemas ambientales empiezan a aparecer y a relacionarse tanto con la industrialización como con la densidad y la existencia de grandes conurbaciones, ${ }^{29}$ todos los indicadores de sostenibilidad aplicados a las zonas urbanas, dan un resultado alarmante.

Sin embargo, y ya son muchos autores que manifiestan ${ }^{30}$, que la ciudad no es el problema de sostenibilidad, sino que hay que entenderla como la gran solución . La existencia de concentración de personas, actividades y funciones, debe ser ahora abordada desde otra perspectiva nueva, desde la óptica de la sostenibilidad, y desde este marco es desde donde hay que empezar a actuar de otra forma para detener primero las tendencias negativas y reconducirlas después, hacia los nuevos objetivos

\footnotetext{
${ }^{26}$ Higueras, E. (2012) Manuales de urbanismo bioclimático para Vitoria-Gasteiz http://www.vitoriagasteiz.org/we001/was/we001Action.do?idioma=es\&aplicacion=wb021\&tabla=contenido\&uid=u6fa2fe e7 14273f1b7eb 7fb5

27 Fariña , (2014) "Los edificios de gran altura afectan particularmente al bienestar mental de madres con niños pequeños y, posiblemente, al de los propios niños" El blog de Jose Fariña.

28 FARIÑA TOJO, J. (1998) La ciudad y el medio natural. Akal. Madrid

${ }^{29}$ El consumo de energía en los edificios residenciales y comerciales representa aproximadamente el $40 \%$ del consumo total de energía final y el 36\% de las emisiones totales de $\mathrm{CO}_{2}$ de la Unión Europea (2008). Fuente UE.
} 
consensuados internacionalmente, Jenks \& Jones (2012) afirman: "Overall, research indicates that there are unlikely to be single spatial or physical solutions, rather that there may be many forms that can achieve sustainability, depending on the context in which they are applied (e.g. Guy and Marvin, 2000; Jenks and Dempsey, 2005). ${ }^{31}$

\begin{tabular}{|c|c|c|c|c|c|c|}
\hline \multirow[b]{2}{*}{$\begin{array}{l}\text { ENFEMEDADES } \\
\text { URBANAS } \\
\text { (CITY } \\
\text { DISEASES) }\end{array}$} & \multicolumn{6}{|c|}{$\begin{array}{l}\text { VARIABLES A CONSIDERAR EN LA PLANIFICIACION Y EL DISEÑO URBANO } \\
\text { (SPATIAL PLANNING AND URBAN DESIGN) }\end{array}$} \\
\hline & $\begin{array}{l}\text { Alta densidad } \\
\text { (High density) }\end{array}$ & \begin{tabular}{|l|} 
Estructura \\
urbana \\
(Urban Structure)
\end{tabular} & $\begin{array}{l}\text { Isla urbana de } \\
\text { calor } \\
\text { (Urban Heat } \\
\text { Island) }\end{array}$ & \begin{tabular}{|l} 
Escasez de \\
zonas verdes \\
(Few green \\
areas)
\end{tabular} & \begin{tabular}{|l} 
Distribución de \\
los usos del \\
suelo \\
(Land use \\
pattern) \\
\end{tabular} & \begin{tabular}{|l|} 
Acabados \\
superficiales \\
(Pavements)
\end{tabular} \\
\hline $\begin{array}{l}1^{0} .- \text { Obesidad y } \\
\text { enfermedades } \\
\text { cardiovasculares } \\
\text { (Obesity and } \\
\text { cardiovascular } \\
\text { diseases) }\end{array}$ & & & & & & \\
\hline $\begin{array}{l}2^{0} .- \\
\text { Enfermedades } \\
\text { respiratorias } \\
\text { (Respiratory } \\
\text { diseases) }\end{array}$ & & & & & & \\
\hline $\begin{array}{l}3^{0} \text {.- Stress } \\
\text { térmico por frio o } \\
\text { calor } \\
\text { (Excess winter } \\
\text { and summer } \\
\text { mortality) }\end{array}$ & & & & & & \\
\hline $\begin{array}{l}4^{0} \text {.- } \\
\text { Enfermedades } \\
\text { especificas de la } \\
\text { infancia y } \\
\text { ancianidad } \\
\text { (Children and } \\
\text { eldery risks) }\end{array}$ & & & & & & \\
\hline
\end{tabular}

Fig. 1. Sombreada la relación entre las principales variables de la planificación y el diseño urbano con las enfermedades, ordenadas por importancia.

Fuente: Adaptación de Delivering Healthier Communities in London 2007.

El reto es grande, debido principalmente a que no nos sirven ni las herramientas ni las soluciones del siglo XX para resolver el problema de la sostenibilidad urbana. La necesidad de establecer acciones transversales, integradas, multidisciplinares, sintéticas y ecosistémicas establece unas nuevas reglas de juego en el que hasta ahora, estaba todo fragmentado, aislado y autónomo ${ }^{32}$. Es por esto que la aportación del barrio saludable, puede convertirse en una nueva vía de transformación de lo

31 Mike Jenks · Colin Jones, Editors Dimensions of the Sustainable City. ISBN 978-1-4020-8646-5(HB) e-ISBN 978-1-4020-8647-2; ISBN 978-1-4020-8645-8(PB)- DOI 10.1007/978-1-4020-8647-2 .Springer Dordrecht Heidelberg London New York

32 Fariña Tojo, J. Merino Merino, B. Cuadernos del OSE sobre políticas de salud en la UE. Número 7: Urbanismo y Salud Pública. Granada: Observatorio de Salud en Europa de la Escuela Andaluza de Salud Pública; García-Sánchez, I. Editora. Diciembre 2012 
existente en un nuevo barrio con soluciones reales y oportunas. La escala del barrio es lo suficientemente compleja para abordar soluciones urbanas, pero a su vez es lo suficientemente limitada para establecer planteamientos eficaces y sencillos. Ya son numerosas las guías en las que se ha manifestado la relación entre el diseño de un barrio y la salud de sus residentes ${ }^{33}$ En el conocimiento de estas influencias y el planteamiento de las nuevas soluciones, no debe olvidar que deben combinarse con otras estrategias institucionales, sociales, económicas y urbanas para que la ciudad se transforme verdaderamente en algo sostenible en este siglo XXI y mejore las condiciones de salud urbana de los ciudadanos. ${ }^{34}$

\section{¿Qué es un barrio saludable?}

Se define barrio saludable, aquel perteneciente a la ciudad consolidada existente, y por lo tanto con importantes deficiencias en cuestiones de eficiencia energética, y con gran contaminación ambiental, que se transformará mediante la adopción de medidas urbanas y arquitectónicas en una nueva realidad, que reduzca su consumo de energías contaminantes y sea capaz de presentar un balance mas equilibrado o incluso positivo de generación de energía limpia para sus actividades y usos, y donde la mayor parte de sus residentes reduzcan las patologías relacionadas con la ciudad industrializada: enfermedades cardiovasculares, respiratorias, de estress térmico o de estrés psicológico (Figura 1). ${ }^{35}$

En las últimas décadas del siglo XX, han aparecido los ecobarrios, como el paradigma del desarrollo sostenible. Sin quitar importancia a estas propuestas, es necesario hacer las siguientes consideraciones. En primer lugar, los ecobarrios representan extraordinarios ejemplos de una nueva forma de hacer tanto arquitectura como urbanismo desde los criterios de sostenibilidad, pero son solo una "gota en el océano"; es decir, representan acciones aisladas, de pequeña escala y en casi todos los casos conocidos, se muestran autónomos e independientes de la ciudad existente 36. No se pueden ver como la solución al problema de sostenibilidad del planeta ${ }^{37}$, ni tampoco servirán para mejorar las condiciones de salud urbana; pero sí se puede extraer de ellos algunas líneas de acción y estrategias que se pueden aplicar en otros contextos y escalas. Creo que es desde esta reflexión, donde aparece la oportunidad de los ecobarrios que merecen un estudio pormenorizado de sus soluciones para buscar la replica en la ciudad consolidada que es donde verdaderamente debemos resolver los problemas actuales. En segundo lugar, la eficiencia de los ecobarrios se

33 Delivering Healthier Communities in London (2007). NHS London Health Urban Development Unit Land Use Consultants and CREH, Capitulo 3 "Health and Planning"

34 Sandin-Vazquez y Sarria-Santamera: "Evaluación de impacto en salud: valorando la efectividad de las políticas en la salud de las poblaciones. " Junio 2008, Revista Española de Salud Pública, no 3. Y Observatorio de salud y Medio Ambiente de Andalucía: Urbanismo, Medio ambiente y salud, ISBN-97884-694-5935-5, Capitulo 5

35 Thomson H, Thomas S, Sellstrom E, Petticrew M, (2013) "Housing improvements for health and associated socio-economic outcomes". 2013 The Cochrane Database of Systematic Reviews. Issue 2 . Collaboration Published by John Wiley \&sons, Ltd. Su objetivo es: "to assess the health and social impacts on residents following improvements to the physical fabric of housing"

36 Bolund, P. \& Hunhammar, S. (1999) 'Ecosystem services in urban areas'. Ecological Economics, 29, pp.293-301.

37 HOUGH, M. (1998). Naturaleza y ciudad. Planificación urbana y procesos ecológicos. Editorial Gustavo Gili. Barcelona 
basa en la mayor parte de los casos en sistemas con infraestructuras de alta tecnología con elementos de generación de calor distrital, centrales de co-generación o tri-generación, etc. ${ }^{38}$ Estas soluciones están alejadas de la realidad de un barrio convencional a corto o medio plazo, por lo que las acciones deben ir en otras direcciones.

Un Barrio saludable es aquel que tiene las siguientes características básicas:

1. Tiene una concienciación de la población en el ahorro energético de combustibles contaminantes, por lo tanto aparece un consumo responsable para todas sus actividades, y desplazamientos

2. Tiene medidas de acondicionamiento pasivo en los espacios exteriores ${ }^{39}$ con una doble finalidad: por un lado reducir la demanda energética de los inmuebles (en invierno o verano); y por otro lado fomentar las actividades en el espacio público que favorecen la relación social y la cohesión del barrio ${ }^{40}$

3. Tiene medidas de acondicionamiento pasivo en las edificaciones. Aparece una diferenciación del diseño de las fachadas según cada orientación ${ }^{41}$ : aparece un diseño de huecos acorde con las necesidades de captación solar o iluminación de cada estancia; tiene un diseño especifico de elementos volados del inmueble según la cantidad de radiación solar que recibe: aparece una diferenciación entre las plantas altas y las bajas del inmueble, etc.

4. Genera una fracción de energía renovable solar limpia para los consumos de los residentes, bien individualmente o bien en una red común para el abastecimiento de servicios comunitarios (ascensores, bombas, alumbrado general, etc) ${ }^{42}$

5. Los residentes reducen sus riesgos frente a enfermedades cardiovasculares, ya que se mueven andando por la proximidad de actividades y servicios del barrio; usan la bicicleta para sus desplazamientos cotidianos ya que existe una red de carriles bici adecuada y segura. ${ }^{43}$

6. Los residentes reducen sus riesgos frente a enfermedades respiratorias, ya que se ha reducido la presencia de contaminantes atmosféricos, especialmente el dióxido de carbono, el plomo, el oxido de nitrógeno y las partículas en suspensión.

\footnotetext{
38 Stritzky, J. von y Cabrerizo, C (2011): Ideas para las ciudades inteligentes del futuro.

39 ERELL, Evyatar; PEARLMUTTER, David; WILLIAMSON, Terry. (2010) Urban microclimate : designing the spaces between buildings. London, etc.: Earthscan

40 HIGUERAS, E. Urbanismo bioclimático. (2006) Editorial Gustavo Gili. $1^{a}$ edición. ISBN-13: 978-84252-2071-5

41 IDAE (Instituto para la Diversificación y el Ahorro de la Energía) (2010): "Prestaciones medias estacionales de equipos y sistemas de producción de frío y calor en edificios de viviendas"

42 AAVV. 2004. RUROS, Designing Open Spaces in the Urban Environment: A Bioclimatic Approach. (2009) Director Marianela Nikolopoulou, Center for Renewable Energy Sources, Department of Buildings. Athens. Greece. ISBN- 960-86907-2-2

43 HIGUERAS , E. El reto de la ciudad habitable y sostenible. (2009) Editorial DAPP.ISBN-978-84-9250719-1
} 
Especialmente las poblaciones más vulnerables, los niños y los ancianos. ${ }^{44}$ Gracias a la distribución de usos del suelo, densidad, movilidad sostenible, control de contaminantes e incremento de las zonas verdes del barrio (tanto en espacios libres, públicos o privados, como en la edificación, cubiertas y fachadas)

7. Los residentes reducen sus riesgos frente a enfermedades mentales ${ }^{45}$ ya que el barrio está equilibrado, es dinámico, está cohesionado y aparece una comunidad estable para afrontar retos y plantear soluciones ante cualquier eventualidad. ${ }^{46}$

Establecidos los retos de la sostenibilidad de los barrios como primer paso para la sostenibilidad urbana, cabe preguntarse si estamos realmente resolviendo los problemas detectados. Las últimas décadas del siglo XX y primera del siglo XXI, se han caracterizado por la existencia de una gran cantidad de diagnósticos, mediante nuevas herramientas como son los indicadores (entre las más innovadoras), que han estructurado los cambios necesarios en los ámbitos medioambiental, social y económico.

Ahora desde la perspectiva de la salud de los residentes de la ciudad, es necesario incorporar las acciones sobre la distribución de los usos del suelo, la movilidad, la reducción del consumo de agua, energía y suelo, la reducción de la demanda energética mediante técnicas adecuadas de acondicionamiento pasivo de las estructuras urbanas y arquitectónicas, y desde luego mediante una eficaz red del sistema verde local y metropolitano, que mitigue las enfermedades de las ciudades, sobre todo hacia las poblaciones de mayor riesgo como son los niños y los ancianos. De esta forma se conseguirán barrios con población más sana y con menores riesgos de salud procedentes de su entorno.

\footnotetext{
44 Declaración de la OMS Parma 2010 sobre Medio Ambiente y Salud: el proyecto aborda las recomendaciones sobre cuestiones ambientales y de salud que afectan el estado de salud de los niños. 45 Wolf, S., Bruhn, JG. The power of clan. The influence of human relationships on heart disease. (1993) New Bruswick, NJ: Transaction Publishers.

46 Bonaiuto, M., Aiello, A., Perugini, M., Bonnes, M., and Ercolani, A.P. (1999) 'Multidimensional Perception of Residential Environment Quality and Neighbourhood Attachment in the Urban Environment'. Journal of Environmental Psychology, 19, pp.331-352
} 